\title{
ON A THEOREM OF H. HOPF
}

\section{TAKIS SAKKALIS}

\section{Department of Mathematical Sciences}

New Mexico State University

Las Cruces, New Mexico 88003

(Received February 1, 1989 and in revised form March 9, 1990)

Abstract. A simple proof of a theorem of $\mathrm{H}$. Hopf [1], via Morse theory, is given.

Key Words and Phrases. Hypersurface, Morse function, critical point, Gauss map, degree.

1980 Ams Subject Classification Codes. 58E05, 55.

1. INTRODUCTION AND THE THEOREM.

Let $f: \mathbf{R}^{n} \rightarrow \mathbf{R}$ be a smooth map, and let

$$
V=\left\{\left(x_{1}, \cdots, x_{n}\right) \in \mathbf{R}^{n} \mid f\left(x_{1}, \cdots, x_{n}\right)=0\right\} .
$$

Suppose $V$ is compact and the gradient, $\nabla f$, of $f$ is nonzero on $V$. Then $V$ in an $(n-1)$ dimensional real orientable hypersurface in $\mathbf{R}^{n}$. Let $U$ be the unbounded component of $\mathbf{R}^{n}-V$. We may suppose that $f>0$ on $U$, otherwise consider $-f$. We shall give $V$ the following orientation. Let $v \in V$ and let $v_{1}, \cdots, v_{n-1}$ be a positively oriented basis for the tangent space $T V_{v}$, regarded as a subspace of $T \mathbf{R}_{v}^{n}$. We say that $V$ has the positive orientation at $v$ if

$$
\operatorname{det}\left[\begin{array}{c}
\nabla f(v) \\
v_{1} \\
\vdots \\
v_{n-1}
\end{array}\right]>0
$$

$V$ has the positive orientation, if it has the positive orientation at each of its points. Let $S^{n-1}$ be the unit sphere in $\mathbf{R}^{n}$, along with its usual orientation. Consider the Gauss map $\eta: V \rightarrow S^{n-1}$ which assigns to each point of $V$, the unit normal vector $\nabla f /\|\nabla f\|$. Let $d$ be the degree of $\eta$. For a real compact manifold $W$, let $\chi(W)$ denote its Euler characteristic. We can now state the theorem which relates $d$, with the Euler characteristic of certain hypersurfaces arising from $f$.

Theorem (Hopf [1]). Let $f, V, d$ be as above. Then

$$
d= \begin{cases}\frac{\chi(V)}{2} & \text { if } n \text { is odd } \\ \chi(f \leq 0) & \text { if } n \text { is even. }\end{cases}
$$




\section{Preliminaries.}

The main idea of the proof of the theorem is to apply Morse theory on $V$, using a convenient Morse function. According to a theorem of Sard, the set of critical values of $\eta$ has measure zero in $S^{n-1}$ [2]. Hence, after rotating the axis if necessary, we may assume that the points $(0, \cdots, 0, \pm 1)$ are not critical values of $\eta$. Let $\pi\left(x_{1}, \cdots, x_{n}\right)=x_{n}$ be the projection onto the last coordinate, and let $h=\left.\pi\right|_{V}$ be the height function on $V$. Let $p$ be a critical point of $h$. At $p$ we have:

$$
f=0, \quad \frac{\partial f}{\partial x_{\imath}}=0, \quad i=1, \cdots, n-1, \quad 1=\lambda \frac{\partial f}{\partial x_{n}}, \quad \lambda \in \mathbf{R} .
$$

LEMma 2.1 [3]. With the above considerations, $p$ is not a critical point of $\eta$, and $p$ is a nondegenerate critical point of $h$.

Proof. We observe that $\eta(p)=(0, \cdots, 0, \pm 1)$, since $\frac{\partial f}{\partial x_{n}}(p) \neq 0$. Hence, $p$ is not a critical point of $\eta$. In terms of local coordinates $u_{1}, \cdots, u_{n-1}$ on $V$, this means that the matrix $\left[\frac{\partial \eta_{1}}{\partial u_{j}}\right], i, j<n$, is nonsingular at $p$. In fact, near $p$ we can choose local coordinates $u_{1}, \cdots, u_{n-1}$ so that $x_{1}=u_{1}, \cdots, x_{n-1}=u_{n-1}, x_{n}=h\left(u_{1}, \cdots, u_{n-1}\right)$. Then,

$$
\eta\left(u_{1}, \cdots, u_{n-1}\right)= \pm\left(\frac{\partial h}{\partial u_{1}}, \cdots, \frac{\partial h}{\partial u_{n-1}},-1\right) / \sqrt{1+\sum_{\jmath=1}^{n-1}\left(\frac{\partial h}{\partial u_{\jmath}}\right)^{2}} .
$$

Hence, $\frac{\partial \eta_{i}}{\partial u_{j}}= \pm \frac{\partial^{2} h}{\partial u_{1} \partial u_{j}}$ at $p$. Therefore, the matrix $\left[\frac{\partial^{2} h}{\partial u_{1} \partial u_{j}}\right], i, j<n$, is nonsingular, which implies that $p$ is a nondegenerate critical point of $h$.

Set $S=\eta^{-1}(0, \cdots,-1), N=\eta^{-1}(0, \cdots, 1)$. Then the above Lemma shows that $h$ is a Morse function on $V$ with critical set $S \cup N$. For $p \in S \cup N$, we denote by $i(p)$ the Morse index of $h$ at $p$, which is equal to the number of negative eigenvalues, multiplicities counted, of the real symmetric matrix $\left[\frac{\partial^{2} h}{\partial u_{i} \partial u_{j}}\right][4]$.

Also, for $p \in S \cup N$ we define $\operatorname{sgn}(p)$ to be

$$
\operatorname{sgn}(p)= \begin{cases}1 & \text { if near } p, \eta \text { preserves the orientation } \\ -1 & \text { if near } p, \eta \text { reverses the orientation }\end{cases}
$$

In addition, if $a$ is a real number, $a \neq 0$, we will denote its signature by $\operatorname{sign}(a)$.

REMARK 2.1. $2 d=\sum_{p \in S \cup N} \operatorname{sgn}(p), \quad \chi(V)=\sum_{p \in S \cup N}(-1)^{\mathfrak{\imath}(p)},[4]$.

We will now compute $\operatorname{sgn}(p)$, for $p \in S \cup N$. Let $G: U \rightarrow V$ be a local parametrization of $V$ near $p$, defined by $G\left(x_{1}, \cdots, x_{n-1}\right)=\left(x_{1}, \cdots, x_{n-1}, h\left(x_{1}, \cdots, x_{n-1}\right)\right)$. Set $\bar{p}=\left(p_{1}, \cdots, p_{n-1}\right)$. Then, 


$$
\operatorname{sgn} d G_{\bar{p}}=\operatorname{signdet}\left[\begin{array}{c}
\nabla f(p) \\
d G\left(\frac{\partial}{\partial x_{1}}\right) \\
\vdots \\
d G\left(\frac{\partial}{\partial x_{n-1}}\right)
\end{array}\right]=(-1)^{n-1} \operatorname{sign} \frac{\partial f}{\partial x_{n}}(p) \text {. }
$$

On the other hand, if $k: U_{1} \rightarrow S^{n-1}$ is a local parametrization of $S^{n-1}$ near the point $\left(0, \cdots, 0, \frac{\partial f}{\partial x_{n}}(p) /\left|\frac{\partial f}{\partial x_{n}}(p)\right|\right)$, defined by $k\left(s_{1}, \cdots, s_{n-1}\right)=$ $\left(s_{1}, \cdots, s_{n-1}, \operatorname{sign} \frac{\partial f}{\partial x_{n}}(p) \sqrt{1-\sum s_{\jmath}^{2}}\right)$, then,

$$
\operatorname{sgn} d k_{0}=\operatorname{sign} \operatorname{det}\left[\begin{array}{c}
\nabla f(p) \\
d k\left(\frac{\partial}{\partial s_{1}}\right) \\
\vdots \\
d k\left(\frac{\partial}{\partial s_{n-1}}\right)
\end{array}\right]=(-1)^{n-1} \operatorname{sign} \frac{\partial f}{\partial x_{n}}(p) \text {. }
$$

Also, near $p, \eta=-\operatorname{sign} \frac{\partial f}{\partial x_{n}}(p) \frac{(\nabla h,-1)}{\sqrt{1+\sum\left(\frac{\partial h}{\partial u}\right)^{2}}}$. Hence,

$$
\operatorname{sgn}(p)=\operatorname{sgnd}\left(k^{-1} \circ \eta \circ G\right)(\bar{p})=\left(-\operatorname{sign} \frac{\partial f}{\partial x_{n}}(p)\right)^{n-1} \cdot \operatorname{sign} \operatorname{det}\left[\frac{\partial^{2} h}{\partial u_{1} \partial u_{\jmath}}\right] \text {. }
$$

Lemma 2.2. For $p \in S \cup N, \operatorname{sgn}(p)=-\operatorname{sign} \operatorname{det} B H(f)(p)$, where $B H(f)=$ $\left[\begin{array}{cc}0 & \nabla(f) \\ \nabla^{t} f & H(f)\end{array}\right]$, is the Bordered Hessian matrix of $f$.

Proof. We have $f\left(u_{1}, \cdots, u_{n-1}, h\left(u_{1}, \cdots, u_{n-1}\right)\right)=0$, where $u_{1}, \cdots, u_{n-1}, h$, are as in Lemma 2.1. By differentiating the above identity twice, and evaluating at $p$, we get

$$
\frac{\partial^{2} f}{\partial x_{i} \partial x_{j}}+\frac{\partial f}{\partial x_{n}}(p) \frac{\partial^{2} h}{\partial u_{i} \partial u_{j}}=0, \quad 1 \leq i, j \leq n-1 .
$$

Using (2. 1) we get $\operatorname{sgn}(p)=\left(-\operatorname{sign} \frac{\partial f}{\partial x_{n}}(p)\right)^{n-1} \cdot \operatorname{sign} \operatorname{det}\left[\frac{\partial^{2} h}{\partial u_{1} \partial u_{1}}\right]=$ sign $\operatorname{det}\left[\frac{\partial^{2} f}{\partial x_{i} \partial x_{j}}\right]=-\operatorname{sign} \operatorname{det} B H(f)(p)$.

REMARK 2.2. If $n$ is even, then $\chi(V)=0$.

Proof. We have $\chi(V)=\sum_{p \in S}(-1)^{i(p)}+\sum_{p \in N}(-1)^{i(p)}$. But if $p \in S$ then $\operatorname{sgn}(p)=(-1)^{i(p)}$, while if $p \in N, \operatorname{sgn}(p)=(-1)(-1)^{i(p)}$. Hence,

$$
\chi(V)=\sum_{p \in S} \operatorname{sgn}(p)-\sum_{p \in N} \operatorname{sgn}(p)=d-d=0 .
$$


3. Proof of the Theorem . Case i. $n$ is odd. We observe from (2. 1), that

$$
\begin{aligned}
\operatorname{sgn}(p)=\operatorname{signdet}\left[\frac{\partial^{2} h}{\partial u_{i} \partial u_{2}}\right] & =(-1)^{i(p)} \text {. Hence, by Remark 2. 1, } \\
\chi(V) & =\sum_{p \in S \cup N}(-1)^{i(p)}=\sum_{p \in S \cup N} \operatorname{sgn}(p)=2 d .
\end{aligned}
$$

Case ii. $n$ is even. Then, let us consider $V^{-}=\{f \leq 0\}$. This is a compact orientable manifold with boundary $V$. Consider the double covering $W$ of $V^{-}$, ramified along $V$, which is defined by

$$
W=\left\{\left(x_{1}, \cdots, x_{n}, x_{n+1}\right) \in \mathbf{R}^{n+1} \mid f\left(x_{1}, \cdots, x_{n}\right)+x_{n+1}^{2}=0\right\} .
$$

$W$ is a compact $n$-dimensional nonsingular hypersurface and $\chi(W)=2 \chi\left(V^{-}\right)-\chi(V)=$ $=2 \chi\left(V^{-}\right)$, since $n$ is even. We orient $W$ as we oriented $V$. On $W$ we consider the height function $\bar{h}$, where $\bar{h}=\left.\bar{\pi}\right|_{W}, \bar{\pi}\left(x_{1}, \cdots, x_{n}, x_{n+1}\right)=x_{n}$. Let $\bar{\eta}: W \rightarrow S^{n}$ be the Gauss map, and let $\bar{d}$ be its degree. Regard $\mathbf{R}^{n}, S, N$ as subsets of $\mathbf{R}^{n+1}$.

As in Lemma 2.1, we have that if $p \in S \cup N$, then $p$ is a nondegenerate critical point of $\bar{h}$. In fact, $S \cup N$ is the critical set of $\bar{h}$, and the points $(0, \cdots, 0, \pm 1,0)$ are not critical values of $\bar{h}$. Let now $p \in S \cup N$. $p$ is viewed as a critical point of both $h$ and $\vec{h}$, and also as a noncritical point of $\eta$ and $\bar{\eta}$. Denote by $\overline{s g n}(p)$, the $\operatorname{sgn}(p)$ viewed as a noncritical point of $\bar{\eta}$. We have:

$$
\operatorname{sgn}(p)=(-1) \operatorname{signdet}\left[\begin{array}{cc}
0 & \nabla f \\
\nabla^{t} f & H(f)
\end{array}\right]=(-1) \operatorname{sign} \operatorname{det}\left[\begin{array}{ccc}
0 & \nabla f & 0 \\
\nabla^{t} f & H(f) & 0 \\
0 & 0 & 2
\end{array}\right]=\overline{\operatorname{sgn}(p)} .
$$

Hence, $d=\bar{d}=\frac{\chi(W)}{2}=\chi\left(V^{-}\right)=\chi(f \leq 0)$. The proof of the theorem is now complete.

\section{REFERENCES}

1. HOPF, H. Über die Curvatura integra geschlossener Hyperflächen, Mathematische Annalen 95, (1925-26), 340-365.

2. SARD, A. The measure of the critical values of differentiable maps, Bull. Amer. Math. Soc. $\underline{48}$ (1942), 883-897.

3. MiLnor, J. On the Betti numbers of real varieties, $\underline{\text { P. AMS }} \underline{15}$ (1964), 275-280.

4. Milnor, J. Morse Theory, Annals of Math. Studies, Princeton University Press, \# 51. 


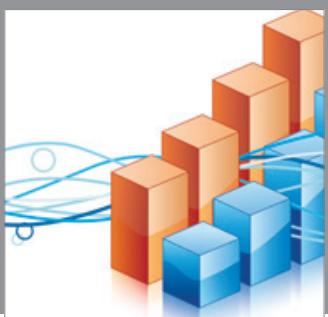

Advances in

Operations Research

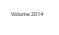

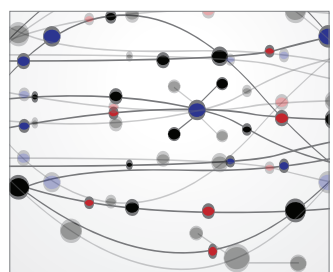

\section{The Scientific} World Journal
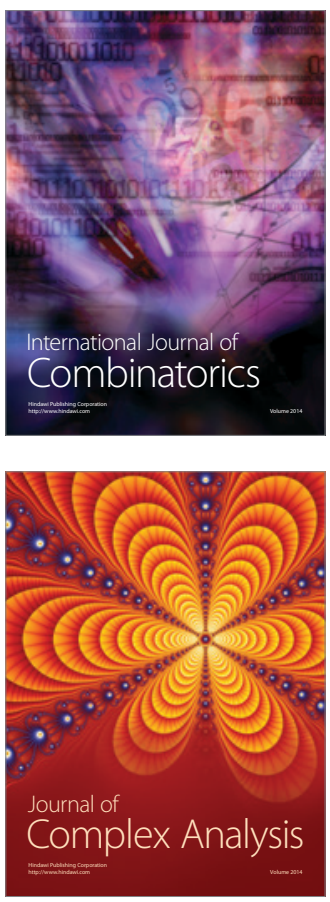

International Journal of

Mathematics and

Mathematical

Sciences
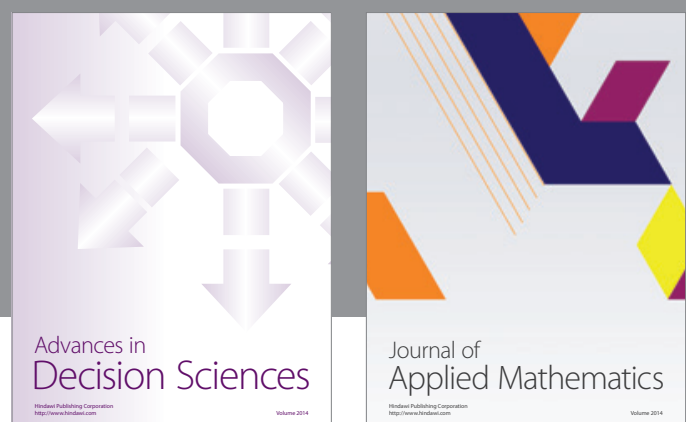

Journal of

Applied Mathematics
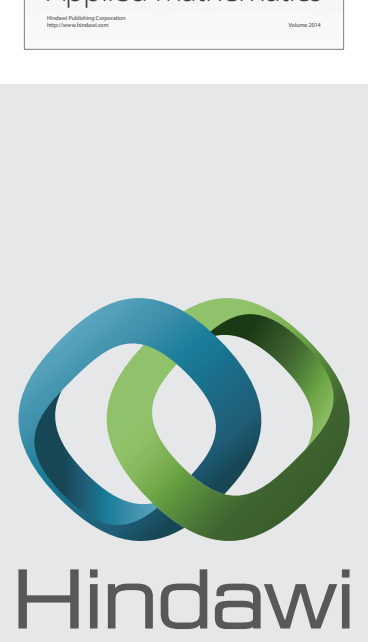

Submit your manuscripts at http://www.hindawi.com
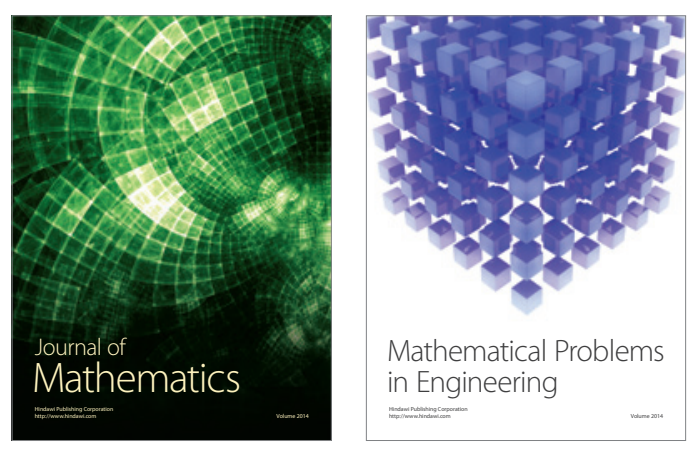

Mathematical Problems in Engineering
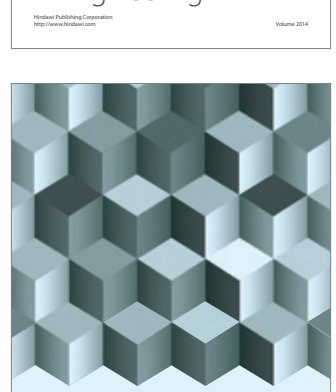

Journal of

Function Spaces
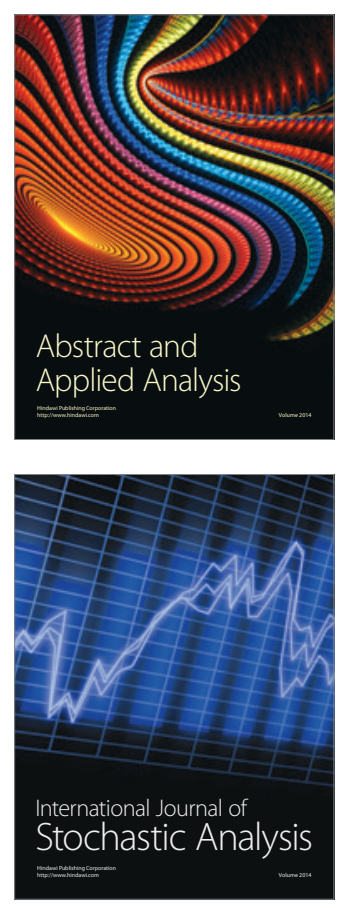

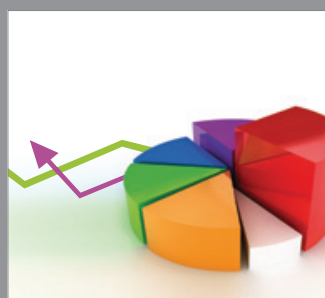

ournal of

Probability and Statistics

Promensencen
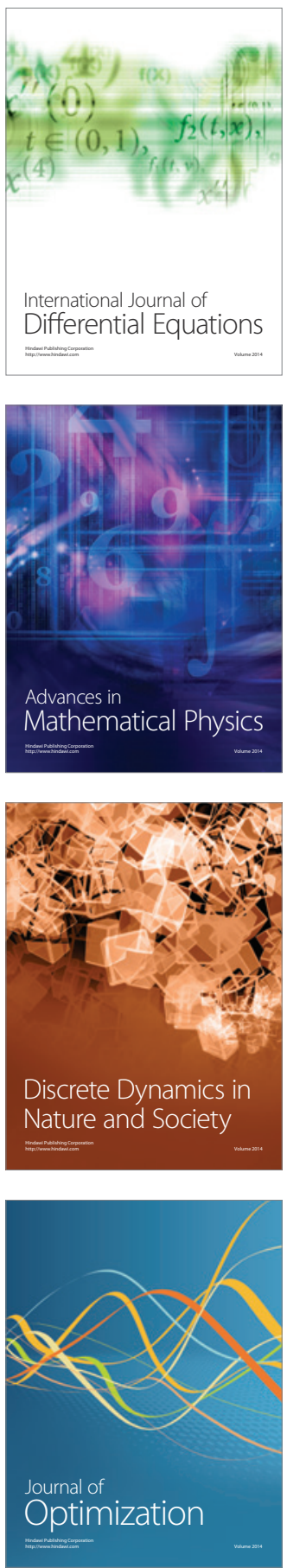